\title{
A Family of 4-Point $n$-Ary Interpolating Scheme Reproducing Conics
}

\author{
Mehwish Bari, Ghulam Mustafa \\ Department of Mathematics, The Islamia University of Bahawalpur, Bahawalpur, Pakistan \\ Email: ghulam.mustafa@iub.edu.pk,mehwishbari@yahoo.com
}

Received April 16, 2013; revised June 5, 2013; accepted July 5, 2013

Copyright (C) 2013 Mehwish Bari, Ghulam Mustafa. This is an open access article distributed under the Creative Commons Attribution License, which permits unrestricted use, distribution, and reproduction in any medium, provided the original work is properly cited.

\begin{abstract}
The $n$-ary subdivision schemes contrast favorably with their binary analogues because they are capable to produce limit functions with the same (or higher) smoothness but smaller support. We present an algorithm to generate the 4-point $n$-ary non-stationary scheme for trigonometric, hyperbolic and polynomial case with the parameter for describing curves. The performance, analysis and comparison of the 4-point ternary scheme are also presented.
\end{abstract}

Keywords: Interpolation; Non-Stationary; Univariate Ternary Refinement; Continuity; Conic Section

\section{Introduction}

Subdivision is a method for making smooth curves/surfaces, which first emerged an addition of splines to arbitrary topological control nets. Effectiveness of subdivision algorithms, their flexibility and ease make them appropriate for many relative computer graphics applications. The schemes generating curves are considered to be the basic tools for the design of schemes generating surfaces.

A general form of univariate $n$-ary subdivision scheme $S$ which maps a control polygon $f^{k}=\left\{f_{i}^{k}\right\}_{i \in \mathbb{Z}}$ to a refined polygon $f^{k+1}=\left\{f_{i}^{k+1}\right\}_{i \in \mathbb{Z}}$ is defined by

$$
f_{n i+s}^{k+1}=\sum_{j \in \mathbb{Z}} a_{n j+s} f_{i-j}^{k}, \quad S=0,1,2, \cdots, n-1,
$$

where the set $a=\left\{a_{i} \mid i \in \mathbb{Z}\right\}$ of coefficients is called mask of the subdivision scheme. The set of coefficients $a^{k}=\left\{a_{i}^{k}: i \in \mathbb{Z}\right\}$ determines the subdivision rule at level $k$ and is termed as the mask at $k$-th level. If the mask $a^{k}$ is independent of $k$, namely if $a^{k}=a, k \in \mathbb{N}$, the subdivision scheme is called stationary otherwise it is called non-stationary. Sometimes, in computer graphics and geometric modeling, it is required to have schemes to construct circular parts or parts of conics. It seems that (linear) stationary schemes cannot generate conics and non-stationary schemes have such a capability to generate trigonometric polynomials, trigonometric splines and, in particular, circles, ellipses and so on. Such schemes are useful in computer graphics and geometric modeling. Successful efforts have been made to establish approximating and interpolating non-stationary schemes which can provide smooth curves and reproduce circle or some trigonometric curves.

The theoretical bases regarding non-stationary schemes are derived from the analysis of stationary schemes. Jena et al. [1] worked on 4-point binary non-stationary subdivision scheme for curve interpolation. Yoon [2] presented the analysis of binary non-stationary interpolating scheme based on exponential polynomials. Beccari et al. [3] worked on 4-point binary non-stationary uniform tension controlled interpolating scheme reproducing conics. Daniel and Shunmugaraj [4] presented 4-point ternary non-stationary interpolating subdivision scheme. In this paper, we present an algorithm to construct 4-point $n$-ary scheme. For simplicity, we have discussed and analyzed 4-point ternary scheme.

This paper is organized as follows. Section 2 presents the construction of 4-point $n$-ary non-stationary interpolating subdivision schemes. As an example, 4-point ternary scheme is presented in this section. Section 3 provides the smoothness of proposed schemes. In the last section conclusion and visual performance of proposed schemes are presented.

\section{Construction of 4-Point $n$-Ary Scheme}

Here we suggest the following algorithm to construct the non-stationary $n$-ary 4-point $(n=2,3,4, \cdots)$, interpo- 
lating schemes for trigonometric, hyperbolic and polynomial cases.

- Choose interpolating function

$f(x)=a_{0}+a_{1} x+a_{2} \cos x+a_{3} \sin x$, or

$f(x)=a_{0}+a_{1} x+a_{2} \cosh x+a_{3} \sinh x$, or

$f(x)=a_{0}+a_{1} x+a_{2} x^{2}+a_{3} x^{3}$.

- Then define the points $\left\{p_{i}^{k} \mid i \in \mathbb{Z}\right\}$ at level $k$ and get system of linear equations by interpolating.

- The data $p_{i+h}^{k}$ corresponding to the abscissas $x=\frac{h t}{n^{k}}, h=-1,0,1,2$.

- Solve the system of linear equations by any well known method to get the values of unknowns.

- Evaluate the interpolating function $f(x)$ at the grid points $\frac{(r-1) t}{n^{k+1}}: r=2,3, \cdots, n$.

- Define the new points $p_{n i}^{k+1}=p_{i}^{k}$.

- Define the new points

$p_{n i+j}^{k+1}=f\left(\frac{(r-1) t}{n^{k+1}}\right), j=r-1, r=2,3, \cdots, n$, as a linear combination of four consecutive points $p_{i-1}^{k}, p_{i}^{k}$, $p_{i+1}^{k}$ and $p_{i+2}^{k}$.

\section{Ternary 4-Point Interpolating Scheme}

Given a set of control points $P^{k}$ at level $k$, using above algorithm, we define a unified ternary 4-point interpolating scheme that makes a new set of control points $p^{k+1}$ by the rule:

$$
\begin{gathered}
\left\{\begin{array}{l}
p_{3 i}^{k+1}=p_{i}^{k}, \\
p_{3 i+1}^{k+1}=\lambda_{1}^{k} p_{i-1}^{k}+\lambda_{2}^{k} p_{i}^{k}+\lambda_{3}^{k} p_{i+1}^{k}+\lambda_{4}^{k} p_{i+2}^{k}, \\
p_{3 i+2}^{k+1}=\lambda_{4}^{k} p_{i-1}^{k}+\lambda_{3}^{k} p_{i}^{k}+\lambda_{2}^{k} p_{i+1}^{k}+\lambda_{1}^{k} p_{i+2}^{k},
\end{array}\right. \\
\eta_{2}^{k}=\left\{2 \sin \left(2 t / 3^{k}\right)-\operatorname{si}\right. \\
\eta_{3}^{k}=\left\{3 \sin \left(2 t / 3^{k+1}\right)+\right. \\
\eta_{4}^{k}=\left\{-\sin \left(t / 3^{k}\right)+3 \sin \left(t / 3^{k+1}\right)\right\} / \eta^{k}, \\
\eta^{k}=6 \sin \left(t / 3^{k}\right)\left\{\cos \left(t / 3^{k}\right)-1\right\} . \\
p_{3 i}^{k+1}=p_{i}^{k}, \\
p_{3 i+1}^{k+1}=\chi_{1}^{k} p_{i-1}^{k}+\chi_{2}^{k} p_{i}^{k}+\chi_{3}^{k} p_{i+1}^{k}+\chi_{4}^{k} p_{i+2}^{k}, \\
p_{3 i+2}^{k+1}=\chi_{4}^{k} p_{i-1}^{k}+\chi_{3}^{k} p_{i}^{k}+\chi_{2}^{k} p_{i+1}^{k}+\chi_{1}^{k} p_{i+2}^{k},
\end{gathered}
$$

where

$$
\begin{gathered}
\lambda_{1}^{k}=\left\{-2\left(4 v_{k+1}+1\right)\right\} / \lambda_{5}^{k}, \\
\lambda_{2}^{k}=\left\{-4\left(v_{k+1}+1\right)\left(-16 v_{k+1}^{3}+1\right)\right\} / \lambda_{5}^{k}, \\
\lambda_{3}^{k}=\left\{4\left(8 v_{k+1}^{4}+8 v_{k+1}^{3}-2 v_{k+1}+1\right)\right\} / \lambda_{5}^{k}, \\
\lambda_{4}^{k}=\left\{-4\left(v_{k+1}+1\right)\right\} / \lambda_{5}^{k},
\end{gathered}
$$

with

$$
\lambda_{5}^{k}=6\left(4 v_{k+1}^{2}-1\right)\left(2 v_{k+1}+1\right)^{2},
$$

where the parameter $v_{k+1}$ can easily be updated at each subdivision step through following equation

$$
v_{k+1}=\sqrt{\frac{1+v_{k}}{2}}, k=0,1,2, \cdots, v_{0} \in(-1, \infty) .
$$

Therefore, given parameter $v_{k}$, the subdivision rules are achieved by first computing $v_{k+1}$ using Equation (2.2) and then by substituting $v_{k+1}$ into (2.1). As a result, depending on the choice of the parameter, we get different schemes. For $v_{k+1}=\cos \left(t / 3^{k+1}\right), \cosh \left(t / 3^{k+1}\right)$ and 1 in (2.1), we can generate following schemes exact for trigonometric (2.3), hyperbolic (2.4) and polynomial (2.5) respectively.

$$
\left\{\begin{array}{l}
p_{3 i}^{k+1}=p_{i}^{k}, \\
p_{3 i+1}^{k+1}=\eta_{1}^{k} p_{i-1}^{k}+\eta_{2}^{k} p_{i}^{k}+\eta_{3}^{k} p_{i+1}^{k}+\eta_{4}^{k} p_{i+2}^{k}, \\
p_{3 i+2}^{k+1}=\eta_{4}^{k} p_{i-1}^{k}+\eta_{3}^{k} p_{i}^{k}+\eta_{2}^{k} p_{i+1}^{k}+\eta_{1}^{k} p_{i+2}^{k},
\end{array}\right.
$$

where

$$
\eta_{1}^{k}=\left\{-2 \sin \left(t / 3^{k}\right)+3 \sin \left(2 t / 3^{k+1}\right)\right\} / \eta^{k},
$$

$$
\begin{aligned}
& \eta_{2}^{k}=\left\{2 \sin \left(2 t / 3^{k}\right)-\sin \left(t / 3^{k}\right)-6 \sin \left(2 t / 3^{k+1}\right)+3 \sin \left(t / 3^{k+1}\right)\right\} / \eta^{k}, \\
& \eta_{3}^{k}=\left\{3 \sin \left(2 t / 3^{k+1}\right)+\sin \left(2 t / 3^{k}\right)-2 \sin \left(t / 3^{k}\right)-6 \sin \left(t / 3^{k+1}\right)\right\} / \eta^{k},
\end{aligned}
$$

where $\chi_{1}^{k}=\eta_{1}^{k}, \quad \chi_{2}^{k}=\eta_{2}^{k}, \quad \chi_{3}^{k}=\eta_{3}^{k}, \quad \chi_{4}^{k}=\eta_{4}^{k}$, after replacing $\sin$ and $\cos$ functions by $\sinh$ and cosh functions in $\eta_{1}^{k}, \eta_{2}^{k}, \eta_{3}^{k}, \eta_{4}^{k}$.

$$
\left\{\begin{array}{l}
p_{3 i}^{k+1}=p_{i}^{k}, \\
p_{3 i+1}^{k+1}=-\frac{5}{81} p_{i-1}^{k}+\frac{60}{81} p_{i}^{k}+\frac{30}{81} p_{i+1}^{k}-\frac{4}{81} p_{i+2}^{k}, \\
p_{3 i+2}^{k+1}=-\frac{4}{81} p_{i-1}^{k}+\frac{30}{81} p_{i}^{k}+\frac{60}{81} p_{i+1}^{k}-\frac{5}{81} p_{i+2}^{k},
\end{array}\right.
$$

Remark 2.1. The scheme (2.3) and (2.4) can be considered as a non-stationary counterpart of the DD stationary scheme [5] i.e. scheme (2.5) because, the masks of the schemes (2.3) and (2.4) converge to the mask of scheme (2.5): 


$$
\begin{aligned}
& \eta_{1}^{k}=\chi_{1}^{k} \rightarrow-\frac{5}{81}, \eta_{2}^{k}=\chi_{2}^{k} \rightarrow \frac{60}{81}, \eta_{3}^{k}=\chi_{3}^{k} \rightarrow \frac{30}{81}, \\
& \eta_{4}^{k}=\chi_{4}^{k} \rightarrow-\frac{4}{81}, \text { as } k \rightarrow \infty .
\end{aligned}
$$

\section{Smoothness Analysis}

The subdivision scheme given in the previous subsection, the coefficients $\left\{\lambda_{i}^{k}\right\}_{i=1,2,3,4}$ in (2.1) may vary from one refinement level to another. Hence the scheme is nonstationary and its smoothness properties can be derived by asymptotical equivalence [6] with the corresponding stationary scheme. Two subdivision schemes $S_{a^{k}}$ and $S_{a}$ are said asymptotically equivalent if

$\sum_{k \in \mathbb{Z}^{+}}\left\|S_{a^{k}}-S_{a}\right\|<+\infty$. In particular, our analysis is based on the generalization of Theorem 8 in [6] to ternary subdivision.

Since our schemes (2.3) and (2.4) are non-stationary then we can use the theory of asymptotic equivalence and generating function formalism [7] to investigate the smoothness of the schemes. First, we need some estimates of $\eta_{i}^{k}$ and $\chi_{i}^{k}, i=1,2,3,4$, which are specified in subsequent lemmas.

\section{Lemma 3.1.}

The mask of scheme (2.3) satisfies following inequalities for sufficiently large $k$.
1) $-\frac{1}{3} \leq \eta_{1}^{k} \leq \frac{2}{3}$
2) $-\frac{4}{3} \leq \eta_{2}^{k} \leq 1$,
3) $-\frac{10}{27} \leq \eta_{3}^{k} \leq \frac{4}{3}$,
4) $-\frac{1}{6} \leq \eta_{4}^{k} \leq \frac{1}{3}$.

Proof. We make use the inequalities $\frac{\sin a}{\sin b} \geq \frac{a}{b}$ for $0 \leq a \leq b<\frac{\pi}{2}, \quad \csc \theta<t \csc t$ for $0<\theta<t<\frac{\pi}{2} \quad$ and $\cos x<\frac{\sin x}{x} \quad\left(\right.$ or $\left.\csc x<\frac{1}{x \cos x}\right)$ for $0<x<\frac{\pi}{2}$ to prove the above inequalities:

Since

$$
\begin{aligned}
\eta_{1}^{k} & =\frac{2 \sin \left(t / 3^{k}\right)-6 \sin \left(t / 3^{k+1}\right) \cos \left(t / 3^{k+1}\right)}{12 \sin \left(t / 3^{k}\right) \sin ^{2}\left(t / 2 \cdot 3^{k}\right)} \\
& =\frac{\sin \left(t / 3^{k}\right)}{6 \sin \left(t / 3^{k}\right) \sin ^{2}\left(t / 2 \cdot 3^{k}\right)}-\frac{\sin \left(t / 3^{k}\right) \cos \left(t / 3^{k+1}\right)}{2 \sin \left(t / 3^{k}\right) \sin ^{2}\left(t / 2 \cdot 3^{k}\right)} .
\end{aligned}
$$

Then for $k \rightarrow \infty$

$$
\eta_{1}^{k}=\frac{1-3 \cos \left(t / 3^{k+1}\right)}{6 \sin ^{2}\left(t / 2 \cdot 3^{k}\right)} \geq \frac{1-3 \cos \left(t / 3^{k+1}\right)}{6}=-\frac{1}{3}
$$

and also for $k \rightarrow \infty$, we get

$$
\begin{aligned}
\eta_{1}^{k} & \leq \frac{-2 \sin \left(t / 3^{k}\right)+6 \sin \left(t / 3^{k}\right) \cos \left(t / 3^{k+1}\right)}{-12 \sin \left(t / 3^{k}\right) \sin ^{2}\left(t / 2 \cdot 3^{k}\right)} \\
& =\frac{2\left(2-6 \sin ^{2}\left(t / 2 \cdot 3^{k+1}\right)\right)}{-12 \sin ^{2}\left(t / 2 \cdot 3^{k}\right)}=\frac{2}{3} .
\end{aligned}
$$

This proves 1). The proofs of 2), 3) and 4) are similar.

\section{Lemma 3.2.}

The coefficients in the scheme (2.4) satisfy following inequalities when subdivision level $k \rightarrow \infty$.
1) $-\frac{1}{3} \leq \chi_{1}^{k} \leq 0$,
2) $-\frac{7}{6} \leq \chi_{2}^{k} \leq \frac{7}{6}$,
3) $-\frac{4}{3} \leq \chi_{3}^{k} \leq \frac{4}{3}$
4) $-\frac{1}{6} \leq \chi_{4}^{k} \leq 0$.

Proof. We make use of following inequality of [8]

$$
\frac{1}{\cosh x} \leq \frac{1+\cos x}{2}=\cos ^{2}\left(\frac{x}{2}\right), x \in\left(-\frac{\pi}{2}, \frac{\pi}{2}\right) \text {. }
$$

This claim holds true if the function $f(x)$ is nonnegative on $[0, \pi / 2)$.

Some other inequalities for $0<x<\frac{\pi}{2}$ are

$$
\begin{gathered}
\sinh \left(\frac{2 x}{3^{k+1}}\right) \leq \sinh \left(\frac{x}{3^{k}}\right), \sinh x \geq 0, \\
\frac{1}{\cosh x}<\frac{x}{\sinh x}, \frac{1}{1-\cosh x} \leq 0 .
\end{gathered}
$$

Since

$$
\begin{aligned}
\chi_{1}^{k} & =\frac{-2 \sinh \left(t / 3^{k}\right)+3 \sinh \left(2 t / 3^{k+1}\right)}{6 \sinh \left(t / 3^{k}\right)\left\{\cosh \left(t / 3^{k}\right)-1\right\}} \\
& \geq \frac{-2 \sinh \left(t / 3^{k}\right)}{6 \sinh \left(t / 3^{k}\right)\left\{\cosh \left(t / 3^{k}\right)-1\right\}} .
\end{aligned}
$$

Then for $k \rightarrow \infty$

$$
\begin{aligned}
& \chi_{1}^{k}=-\frac{1}{33\left\{\cosh \left(t / 3^{k}\right)-1\right\}} \geq-\frac{1+\cos \left(t / 3^{k}\right)}{3\left(1-\cos \left(t / 3^{k}\right)\right)} \\
& =-\frac{1+\cos \left(t / 3^{k}\right)}{3\left(2 \sin ^{2}\left(t / 2 \cdot 3^{k}\right)\right)}=-\frac{1}{3},
\end{aligned}
$$


and similarly for $k \rightarrow \infty$

$$
\begin{aligned}
\chi_{1}^{k} & =\frac{-2 \sinh \left(t / 3^{k}\right)+3 \sinh \left(2 t / 3^{k+1}\right)}{6 \sinh \left(t / 3^{k}\right)\left\{\cosh \left(t / 3^{k}\right)-1\right\}} \\
& \leq \frac{3 \sinh \left(t / 3^{k}\right)}{6 \sinh \left(t / 3^{k}\right)\left\{\cosh \left(t / 3^{k}\right)-1\right\}}=0 .
\end{aligned}
$$

This proves 1). The proofs of 2), 3) and 4) are similar.

The following two Lemmas are the consequence of previous Lemmas.

\section{Lemma 3.3.}

1) $\left|\eta_{1}^{k}-\left(\frac{-5}{81}\right)\right| \leq \frac{59}{81}$,

2) $\left|\eta_{2}^{k}-\frac{60}{81}\right| \leq \frac{21}{81}$,

3) $\left|\eta_{3}^{k}-\frac{30}{81}\right| \leq \frac{78}{81}$,

4) $\left|\eta_{4}^{k}-\left(\frac{-4}{81}\right)\right| \leq \frac{31}{81}$.

Proof. Since $\eta_{1}^{k} \rightarrow-\frac{5}{81}$ as $k \rightarrow \infty$ and by 1 ) of

Lemma 3.1, we have 1). Similarly, we get 2), 3) and 4).

\section{Lemma 3.4.}

1) $\left|\chi_{1}^{k}-\left(\frac{-5}{81}\right)\right| \leq-\frac{5}{81}$,

2) $\left|\chi_{2}^{k}-\frac{60}{81}\right| \leq \frac{23}{54}$,

3) $\left|\chi_{3}^{k}-\frac{30}{81}\right| \leq \frac{78}{81}$,

4) $\left|\chi_{4}^{k}-\left(\frac{-4}{81}\right)\right| \leq \frac{4}{81}$.

Proof. Since $\chi_{1}^{k} \rightarrow-\frac{5}{81}$ as $k \rightarrow \infty$ and by 1 ) of

Lemma 3.2, we have 1). Similarly, we get 2), 3) and 4).

\section{Lemma 3.5.}

The Laurent polynomial $\lambda^{k}(z)$ of the $k^{\text {th }}$ level of the scheme $S_{\lambda^{k}}$ defined by (2.1) can be written as

$$
\begin{aligned}
\lambda^{k}(z)= & \left(\frac{1+z+z^{2}}{3}\right) a^{k}(z) \text { where } \\
a^{k}(z)= & 3 \lambda_{4}^{k} z^{5}+3\left(\lambda_{1}^{k}-\lambda_{4}^{k}\right) z^{4}-3 \lambda_{1}^{k} z^{3}+3\left(\lambda_{3}^{k}+\lambda_{4}^{k}\right) z^{2} \\
& +3\left(\lambda_{1}^{k}+\lambda_{2}^{k}-\lambda_{3}^{k}-\lambda_{4}^{k}\right) z+3\left(\lambda_{3}^{k}+\lambda_{4}^{k}\right) \\
& -3 \lambda_{1}^{k} z^{-1}+3\left(\lambda_{1}^{k}-\lambda_{4}^{k}\right) z^{-2}+3 \lambda_{4}^{k} z^{-3} .
\end{aligned}
$$

Proof. By (2.1), we have

$$
\begin{aligned}
\lambda^{k}(z)= & \lambda_{4}^{k} z^{5}+\lambda_{1}^{k} z^{4}+\lambda_{3}^{k} z^{2}+\lambda_{2}^{k} z^{1}+1+\lambda_{2}^{k} z^{-1} \\
& +\lambda_{3}^{k} z^{-2}+\lambda_{1}^{k} z^{-4}+\lambda_{4}^{k} z^{-5} .
\end{aligned}
$$

It can be easily verified that

$$
\lambda^{k}(z)=\left(\frac{1+z+z^{2}}{3}\right) a^{k}(z) \text {. }
$$

\section{Lemma 3.6.}

The stationary scheme $\left\{S_{a}\right\}$ defined by (2.5) associated with the symbol

$$
\begin{gathered}
a(z)=\frac{1}{81}\left\{-4 z^{5}-5 z^{4}+30 z^{2}+60 z^{1}+81+60 z^{-1}\right. \\
\left.+30 z^{-2}-5 z^{-4}-4 z^{-5}\right\}
\end{gathered}
$$

is $C^{1}$.

Proof. To prove that $\left\{S_{a}\right\}$ is $C^{1}$, consider

$$
\begin{aligned}
& b(z)=\frac{3 a(z)}{\left(1+z+z^{2}\right)^{2}} \\
& =\frac{1}{27}\left\{-4 z^{-3}+3 z^{-2}+6 z^{-1}+17+6 z+3 z^{2}-4 z^{3}\right\} .
\end{aligned}
$$

Since

$$
\begin{aligned}
\left\|S_{b}\right\|_{\infty} & =\max \left\{\sum_{j \in \mathbb{Z}}\left|b_{3 j}\right|, \sum_{j \in \mathbb{Z}}\left|b_{3 j+1}\right|, \sum_{j \in \mathbb{Z}}\left|b_{3 j+2}\right|\right\} \\
& =\max \left\{\frac{25}{27}, \frac{9}{27}, \frac{9}{27}\right\}<1
\end{aligned}
$$

then by [[7], corollary 4.11] the scheme $\left\{S_{a}\right\}$ is $C^{1}$.

Lemma 3.7.

The scheme $S_{\lambda^{k}}$ defined by (2.1) is $C^{1}$.

Proof. Since $\left\{S_{a}\right\}$ is $C^{1}$ by Lemma 3.6, in view of [[6], Theorem 8(a)], it is sufficient to show that

$$
\sum_{k=0}^{\infty} 3^{k}\left\|S_{\lambda^{k}}-S_{a}\right\|_{\infty}<\infty
$$

where

$$
\begin{aligned}
& \left\|S_{\lambda^{k}}-S_{a}\right\|_{\infty} \\
& =\max \left\{\sum_{j \in \mathbb{Z}}\left|\lambda_{3 j}^{k}-a_{3 j}\right|, \sum_{j \in \mathbb{Z}}\left|\lambda_{3 j+1}^{k}-a_{3 j+1}\right|, \sum_{j \in \mathbb{Z}}\left|\lambda_{3 j+2}^{k}-a_{3 j+2}\right|\right\} \\
& =\max \left\{\left|-3 \lambda_{1}^{k}-\frac{5}{27}\right|+\left|3 \lambda_{4}^{k}+\frac{4}{27}\right|+\left|3 \lambda_{3}^{k}+3 \lambda_{4}^{k}-\frac{26}{27}\right|,\right. \\
& \left.2\left|3 \lambda_{1}^{k}-3 \lambda_{4}^{k}+\frac{1}{27}\right|+\left|3 \lambda_{1}^{k}+3 \lambda_{2}^{k}-3 \lambda_{3}^{k}-3 \lambda_{4}^{k}-\frac{29}{27}\right|\right\} .
\end{aligned}
$$

Note that

$$
\begin{aligned}
& \left|3 \lambda_{1}^{k}+3 \lambda_{2}^{k}-3 \lambda_{3}^{k}-3 \lambda_{4}^{k}-\frac{29}{27}\right| \\
& \leq\left|\lambda_{1}^{k}-\frac{-5}{81}\right|+\left|\lambda_{2}^{k}-\frac{60}{81}\right|+\left|\lambda_{3}^{k}-\frac{30}{81}\right|+\left|\lambda_{4}^{k}-\frac{-4}{81}\right| .
\end{aligned}
$$

Since $\sum_{k=0}^{\infty} 3^{k}\left|\lambda_{1}^{k}-\left(\frac{-5}{81}\right)\right|<\infty$, 
$\sum_{k=0}^{\infty} 3^{k}\left|\lambda_{2}^{k}-\left(\frac{60}{81}\right)\right|<\infty, \sum_{k=0}^{\infty} 3^{k}\left|\lambda_{3}^{k}-\left(\frac{30}{81}\right)\right|<\infty \quad$ and $\sum_{k=0}^{\infty} 3^{k}\left|\lambda_{1}^{k}+\left(\frac{4}{81}\right)\right|<\infty$, then by Lemma 3.3, it follows that

$$
\sum_{k=0}^{\infty} 3^{k+1}\left|\lambda_{1}^{k}+\lambda_{2}^{k}-\lambda_{3}^{k}-\lambda_{4}^{k}-\frac{29}{81}\right|<\infty
$$

By Lemma 3.3, we can also show that

$$
\begin{aligned}
& \sum_{k=0}^{\infty} 3^{k}\left|-3 \lambda_{1}^{k}-\frac{5}{27}\right|<\infty, \quad \sum_{k=0}^{\infty} 3^{k}\left|3 \lambda_{4}^{k}+\frac{4}{27}\right|<\infty, \\
& \sum_{k=0}^{\infty} 3^{k}\left|3 \lambda_{3}^{k}+3 \lambda_{4}^{k}-\frac{26}{27}\right|<\infty \text { and } \\
& \sum_{k=0}^{\infty} 3^{k}\left|6 \lambda_{1}^{k}-6 \lambda_{4}^{k}+\frac{2}{27}\right|<\infty .
\end{aligned}
$$

Hence $\sum_{k=0}^{\infty} 3^{k}\left\|S_{\lambda^{k}}-S_{a}\right\|_{\infty}$.

\section{Conclusions}

To the aim of reproducing conic sections, we introduce an algorithm for generation of 4-point $n$-ary interpolating scheme. In particular, we define 4-point interpolating scheme that unifies three different curves schemes which are capable of representing trigonometric, hyperbolic and polynomial functions.

The resulting ternary algorithm allows us to efficiently define limit curves that combine all the ingredients of locality, $C^{1}$ smoothness, user-independence, local tension control and reproduction of conics, see Figure 1.

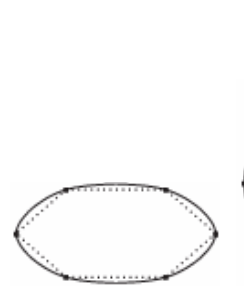

(a)

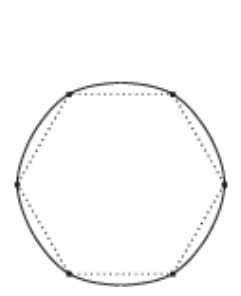

(b)

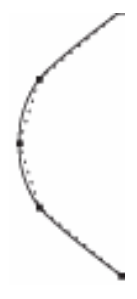

(c)

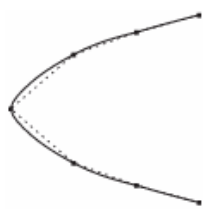

(d)
Figure 1. Dotted lines indicate the initial closed and open polygons. Solid continuous curves are generated by proposed ternary interpolating scheme for trigonometric case.

\section{Acknowledgements}

This work is supported by the Indigenous Ph.D Scholarship Scheme of Higher Education Commission (HEC) Pakistan.

\section{REFERENCES}

[1] M. K. Jena, P. Shunmugaraj and P. C. Das, “A Non-Stationary Subdivision Scheme for Curve Interpolation,” ANZIAM Journal, Vol. 44, No. E, 2003, pp. 216-235.

[2] J. Yoon, "Analysis of Non-Stationary Interpolatory Subdivision Schemes Based on Exponential Polynomials," Geometric Modeling and Processing, Vol. 4077, 2006, pp. 563-570.

[3] C. Beccari, G. Casciola and L. Romani, “A Non-Stationary Uniform Tension Controlled Interpolating 4-Point Scheme Reproducing Conics," Computer Aided Geometric Design, Vol. 24, No. 1, 2007, pp. 1-9.

doi:10.1016/j.cagd.2006.10.003

[4] S. Daniel and P. Shunmugaraj, "Some Interpolating NonStationary Subdivision Schemes," International Symposium on Computer Science and Society, Kota Kinabalu, 16-17 July 2011, pp. 400-403. doi:10.1109/ISCCS.2011.110

[5] G. Deslauriers and S. Dubuc, "Symmetric Iterative Interpolation Processes,” Constructive Approximation, Vol. 5, No. 1, 1989, pp. 49-68. doi:10.1007/BF01889598

[6] N. Dyn and D. Levin, "Analysis of Asymptotically Equivalent Binary Subdivision Schemes,” Journal of Mathematical Analysis and Applications, Vol. 193, No. 2, 1995, pp. 594-621. doi:10.1006/jmaa.1995.1256

[7] N. Dyn and D. Levin, "Subdivision Schemes in Geometric Modelling,” Acta Numerica, Vol. 11, 2002, pp. 73-144. doi:10.1017/S0962492902000028

[8] R. Klen, M. Lehtonen and M. Vuorinen, “On Jordan Type Inequalities for Hyperbolic Functions,” Journal of Inequalities and Applications, Vol. 2010, 2010, Article ID: 362548. 